\title{
EDITORIAL
}

\section{Adherence to treatment of osteoporosis: a need for study}

\author{
F. Lekkerkerker • J. A. Kanis $\cdot$ N. Alsayed $\cdot$ \\ G. Bouvenot $\cdot$ N. Burlet $\cdot$ D. Cahall $\cdot$ A. Chines • \\ P. Delmas $\cdot$ R.-L. Dreiser $\cdot$ D. Ethgen $\cdot$ N. Hughes $\cdot$ \\ J.-M. Kaufman • S. Korte • G. Kreutz • A. Laslop • \\ B. Mitlak • V. Rabenda $\cdot$ R. Rizzoli • A. Santora • \\ R. Schimmer $\cdot$ Y. Tsouderos $\cdot$ P. Viethel $\cdot$ J.-Y. Reginster
}

Received: 18 January 2007 / Accepted: 23 May 2007 /Published online: 22 June 2007

(C) International Osteoporosis Foundation and National Osteoporosis Foundation 2007

\begin{abstract}
Summary Adherence to anti-osteoporosis medications is currently low and is associated with poor anti-fracture efficacy. This manuscript reviews the potential design of clinical studies that aim to demonstrate improved adherence, with new chemical entities to be used in the management of osteoporosis.
\end{abstract}

On behalf of the Group for the Respect of Ethics and Excellence in Science (GREES), section osteoporosis.

\section{F. Lekkerkerker \\ Medicines Evaluation Board, \\ The Hague, The Netherlands}

\section{J. A. Kanis}

University Of Sheffield,

Sheffield, UK

\author{
N. Alsayed \\ Supreme S.A, \\ Liège, Belgium \\ G. Bouvenot \\ French National Authority \\ Paris, France \\ N. Burlet \\ Nyon, Switzerland \\ D. Cahall \\ Sanofi-Aventis, France
A. Chines
Wyeth Research,
Philadelphia, PA, USA
P. Delmas
Hôpital Edouard Herriot,
Lyon, France

for Health-Transparency Committee,

International Osteoporosis Foundation,
Introduction Several medications have been unequivocally shown to decrease fracture rates in clinical trials. However, in real life settings, long-term persistence and compliance to anti-osteoporosis medication is poor, hence decreasing the clinical benefits for patients.

Methods An extensive search of Medline from 1985 to 2006 retrieved all trials including the keywords osteoporosis, compliance, persistence or adherence followed by a

\author{
R.-L. Dreiser \\ Rhumatologie-Hôpital Bichat, \\ Paris, France \\ D. Ethgen \\ Clinical Development, GSK, \\ Philadelphia, PA, USA \\ N. Hughes \\ Amgen Europe GmbH, \\ Zug, Switzerland \\ J.-M. Kaufman \\ UZ Gent, \\ Gent, Belgium \\ S. Korte \\ Novartis Pharma AG, \\ Basel, Switzerland \\ G. Kreutz \\ Council for International Organisations of Medical Sciences, \\ Geneva, Switzerland
}

A. Laslop

Austrian Medicines and Medical Devices Agency,

Vienna, Austria

B. Mitlak

Eli Lilly \& Co,

Indianapolis, IN, USA 
critical appraisal of the data obtained through a consensus expert meeting.

Results The impact of non-adherence on the clinical development of interventions is reviewed, so that clinicians, regulatory agencies and reimbursement agencies might be better informed of the problem, in order to stimulate the necessary research to document adherence.

Conclusion Adherence to therapy is a major problem in the treatment of osteoporosis. Both patients and medication factors are involved. Adherence studies are an important aspect of outcomes studies, but study methodologies are not well developed at the moment and should be improved. Performing adherence studies will be stimulated when registration authorities accept the result of these studies and include the relevant information in Sect. 5.1 of the summary of product characteristics. Reimbursement authorities might also consider such studies as important information for decisions on reimbursement.

Keywords Adherence - Compliance - Persistence . Registration $\cdot$ Reimbursement

\section{Introduction}

Effective treatments for postmenopausal osteoporosis have been available now for more than 10 years. Large wellcontrolled randomized clinical trials (RCTs) have shown that interventions decrease vertebral fracture risk by about $50 \%$. Significant effects of some treatments on non-vertebral fractures have also been shown, though the effect size is often smaller. Despite this clear demonstration of a therapeutic benefit, several observational studies have shown that efficacy is much lower in patients that are incompletely adherent to their therapy. A number of studies have shown

\footnotetext{
V. Rabenda

CHU Sart Tilman,

Liège, Belgium

R. Rizzoli

Centre Collaborateur de l'Oms pour la prevention de

l'osteoporose,

Geneva, Switzerland

A. Santora

Merck \& Co, Inc,

Whitehouse Station, NJ, USA

R. Schimmer

F. Hoffman-La Roche Ltd,

Basel, Switzerland

Y. Tsouderos

Institut de Recherches Internationales Servier,

Paris, France
}

that up to $50 \%$ of patients no longer adhere after the first year of therapy and that poor persistence and compliance are associated with higher fracture rates. Estimates include a $19-36 \%$ higher fracture risk with a compliance of less than $80 \%$ [1-6]. The most detailed analysis described a dosedependency for persistence on fracture risk [2]. Fracture rates were stable until compliance as judged by the medical possession ratio (MPR, see later) fell below about $50 \%$. Similarly, treatment-induced increases in BMD were less in poorly compliant patients. In patients with compliance rates of $<66 \%$, BMD increased by $2.8 \%$ over 1 year at the lumbar spine, whereas BMD increased by $3.8 \%$ in patients with the higher compliance rates. Thus, as noted in a recent comment in the Lancet, compliance with osteoporosis therapy is the weakest link [8].

This phenomenon is not unique for osteoporosis, and it has been shown for other interventions in other chronic diseases such as hypercholesterolaemia, heart diseases as well as for the combined antiretroviral treatment for HIV. In general adherence is a particular problem in chronic asymptomatic conditions $[9,10]$.

Against this background, GREES organized a meeting to discuss the impact of non-adherence on the clinical development of interventions for the management of osteoporosis, so that clinicians, regulatory agencies and reimbursement agencies might be better informed of the problem, to stimulate the necessary research, to document adherence, and, perhaps, to investigate the manner by which adherence could be improved. The meeting was informed by a literature search aimed to identify all papers relating to adherence in the prevention or treatment of osteoporosis. A search of Medline using a predefined strategy was made in September 2006.

An additional search was made of abstracts in relevant journals over the previous three years. No language, date or study-type restrictions were applied to the search. The reference

P. Viethel

Merck-Theramex,

Monaco, Monaco

J.-Y. Reginster

University of Liège,

Liège, Belgium

J.-Y. Reginster $(\bowtie)$

Bone and Cartilage Metabolism Research Unit,

CHU Centre-Ville, Policliniques L. BRULL,

Quai Godefroid Kurth 45 (9ème étage),

4020 Liege, Belgium

e-mail: jyreginster@ulg.ac.be 
lists of relevant articles were hand searched and sifted by title for relevance, and thereafter by the full publication.

\section{Defining adherence}

When studying adherence there is a need for precise definition of the terminology that will be used. In the literature there is a wide variety of definitions. A term commonly used is compliance, but it has been argued that the term implies "obedience". This has resulted in alternative terminology that also infers the active choice of the patient [11, 12], such as adherence [13], patient cooperation [14], therapeutic alliance [15] or concordance [16]; terms that capture the agreement between patient and physician. For these reasons, it is important to provide definitions. The authors favour the use of the terms adherence, persistence and compliance, which are defined below and are in line with the definitions issued by the International Society for Pharmacoeconomic and Outcomes Research (ISPOR).

Adherence General term encompassing all aspects mentioned below.

Persistence For how long the medication is taken. Persistence could be expressed as the number of days until dropout or the proportion of the cohort still on medication after a given time since first prescription. Non-persistence is assumed to be the same as discontinuation if a treatment gap is longer than a set number of days.

Compliance Proximity to the treatment recommendations as given in the official product information (SPC). It is often simplified to mean the number of doses taken divided by the number of prescribed doses. This simplification does not include some important aspects of compliance, such as taking medication with food (for the oral bisphosphonates), at the correct time of the day, too large doses to compensate for forgotten doses, pill dumping, etc.

Primary non-adherence Where a patient is prescribed a drug and then never fills the prescription.

\section{Quantifying adherence}

Adherence is often quantified as medical possession ratios (MPR), which usually is defined as the sum of all days of medication supply received during a given time, say 1 year, divided by the 365 potential days of supply. The value of the MPR is limited in that it does not provide insight into consistency of refilling or whether a drug is being taken as described. The MPR is, in effect, a mixture of persistence and adherence and any improved outcomes associated with a higher MPR may be, to some extent, because those patients take their drugs for a longer time, i.e., are more persistent.

The literature often blurs the distinction between nonpersistence and discontinuation, but the two concepts are not necessarily equivalent. An individual who is non-persistent may have a gap in treatment, but may resume medication at some point in the future. If treatment is stopped, but restarted, a patient may be considered to be non-persistent, depending upon the drug-free interval. What is an acceptable 'grace period' varies between studies and ranges from 2 weeks [17, 18] to 2 months or more [7, 19-21], but is often not specified. In general, the longer the defined grace period, the higher the adherence rates. For example, in a study comparing alendronate and ibandronate, persistence was judged to be $38 \%$ and $56 \%$, respectively, when allowing a two-week grace period, though at 6 months, 73 and $80 \%$ of patients had refilled 5 of the 6 monthly prescriptions [18].

It is also relevant to note that persistence rates depend upon whether a single agent or multiple agents are considered. Persistence with a specific agent does not take account of switching treatments. The available data suggest that a substantial minority of patients switch from one to another agent. Estimates of switching of osteoporosis treatment vary between $11 \%$, and $31 \%$ [17, 22, 23]. These difficulties in the assessment of persistence emphasise the complexity of measuring adherence accurately and argue for a minimum standard for the reporting of adherence.

\section{Pattern of adherence}

The heterogeneity in reporting adherence rates impedes meaningful comparisons between studies. Greater reliance can be placed on comparisons made within studies, for example comparisons of bisphosphonates given daily or weekly.

Adherence typically decreases with time, particularly in the first treatment year. For instance in one study $47 \%$ of the patient did not adhere after 3 months, 70\% after 12 months [7]. For persistence, several long-term studies indicate that this remains stable thereafter for 5 years or more [2, 21]. For compliance, rates fall over two years and then remain stable. The absolute levels of persistence and compliance are more difficult to assess because of differences in reporting. Although several studies show low persistence, in the order of $20-50 \%$, many studies show persistence rates that are rather similar to those reported in randomised controlled trials. In particular, those from the UK show rates on the order of $80 \%[18,22,24,25]$. The lowest rates reported are from North America where in some studies, low persistence may reflect the lack of an 
affordable treatment for some patients, known to be an important factor in non-persistence.

There are fewer studies of compliance and nearly all are from North America. Estimates vary from 47-70\%. Compliance may be higher with weekly compared with daily administration of bisphosphonates [20]. In a large US managed-care database, with a follow-up of up to 5 years, three-quarters of patients had an MPR below $80 \%$ and $53 \%$ had a compliance below 50\% [2]. This figure included, however, patients who became non-persistent. When account is taken of non-persistence, compliance in patients taking medication is higher as judged by MPR $[2,26]$.

\section{The determinants of adherence}

Poor adherence is a particular problem in chronic asymptomatic conditions [9, 27]. The causes for non-adherence are poorly understood; in principle they can be patientdependent (age, education, motivation) or medicationdependent (dosage regime or frequency of administration, special precautions taking the medicine). In addition, the physician-patient relationship, physician training, nurse-led programs and patient education can be important factors in adherence [28, 29]. Few studies have investigated predictors for non-adherence, as has been done for AIDS treatment [30]. Determinants of low persistence and compliance in the context of osteoporosis include side effects, poor general health, the absence of a prior fracture, old age, not being engaged in social activities and fracture early during treatment $[1,2,23,31]$. A major perceived problem with the oral bisphosphonates is the inconvenience of the regimen, and there is a well established association between complex dosing regimens and compliance [32]. A survey in the UK cited inconvenience, the belief that the drug would stop working and concern over drugdependence as major determinants of non-persistence [33]. In multivariate analyses, however, these various factors explain but a minority of non-adherent behaviour [33].

\section{The consequences of poor adherence}

As noted above, the consequences of poor compliance and persistence lie in the reduced effectiveness of treatment but the quantum is insecure. The most detailed analysis described a dose-dependency for persistence on fracture risk [7]. Fracture rates were stable until compliance (excluding non-persistent patients) fell below about 60 to $80 \%$ as judged by the medical possession ratio (MPR) $[2,26]$.

Although, non-adherence appears to be associated with major impact on health outcomes, this is caused only in part by less than optimal medicinal treatment. Non-adherence as such is a risk factor for lower health outcome. For example, a placebo controlled study with amiodarone on cardiac mortality demonstrated that poor adherence to medication has an negative impact on outcome even in the placebo arm [34]. This phenomenon has also been demonstrated in another cardiovascular trial (Charm) with candesartan [35]. In these last two comparative studies, adherence to placebo resulted, respectively, in a lower odds ratio for death or in a lower all cause mortality with a hazard ratio of 0.65 compared to non adherent placebo treated patients. Thus, although these various studies indicate a consistent association between poor compliance and reduced effectiveness of intervention, the association should, however, be cautiously interpreted since non-compliant patients have higher co-morbidity rates, are more frail and have higher health-care expenditure. Some of these studies have 'adjusted' for confounding factors, but information on potential confounders is limited and confined to the few data available from medical claims [1, 2, 21, 31]. It is plausible that poorly compliant patients have, therefore, higher fracture risks before treatment is started. In other words the association may not be causal, or the quantum may be overestimated. Indeed, fracture risks have been reported to be higher and BMD lower in non-persistent patients taking a placebo compared with persistent patients in the placebo wing of an intervention study [31].

\section{Improving adherence}

Adherence to osteoporosis treatment may be influenced by inconvenient dosage requirements, e.g., taking oral bisphosphonates daily at least 30 minutes before breakfast in an upright position. There are several options for improving adherence, e.g., nurse-led follow up, education programs and easier-to-follow medication regimes. The monitoring of treatment may also have a role, and a randomised study has suggested that adherence is improved in women with osteoporosis monitored with the measurement of biochemical indices of bone turnover [36]. In addition, new formulations and dosages schemes have been developed which in principle could help to improve adherence to treatment. For example, a weekly bisphosphonate regimen improves persistence of therapy compared with daily administration [37]. However, even with the weekly regimen, the benefit for adherence remains sub optimal indicating a need to further improve adherence. New options for intermittent treatment are now available, including monthly oral formulations, quarterly intravenous injections, six-monthly injections, and yearly infusions are in development. Weekly and monthly oral bisphosphonate treatment and monthly and yearly intravenous treatment 
have comparable effects on BMD as daily treatment. If these new intermittent treatment regimens are as efficacious and safe as the older regimens, they could have major impact on persistence. When comparing intermittent with non intermittent regimes it should be clearly defined what is meant by intermittent therapy and what is the precise objective of the study. These studies may be difficult to perform; for example, comparing daily oral treatment with yearly intravenous infusion treatment requires a long-term study.

Both health authorities and industry are interested in studies that measure adherence in patient groups taking different formulations and dosing regimens. Adherence could have practical pharmaco-economic consequences and could affect reimbursement decisions. The impact of poor adherence on cost-effectiveness is not straightforward. Whereas poor adherence decreases the effect of intervention, this is offset to some extent by a reduction in the cost of medication. In models of persistence it has been assumed simplisticly that $20-80 \%$ of patients complete a full 5 -year course of treatment, and that the remaining non-persistent patients receive 3 months of drug treatment for no health gain $[38,39]$. The impact on cost-effectiveness was relatively small until persistence fell below about $20 \%$. The problem with this approach is that it does not reproduce the real world. For example, those that discontinue treatment do so at time points throughout the 5-year period and should, thus, receive some health benefit and additional drug costs. More realistic economic models that capture the complex elements of adherence are required. Pharmaco-economic evaluation should not be limited to cost-effectiveness, and social costs are also important determinants. Industry is interested in these issues because better adherence to regimens could also be translated in promotional claims. However, registration authorities will only allow results of adherence studies to be included in the official product information if an effect on efficacy and/or safety has been demonstrated.

\section{How to investigate adherence}

Adherence to therapy is different in clinical trials when patients are intensively followed and treated in a highly structured environment, as found in phase III studied, compared with what occurs in daily clinical practice. Clinical trials will enhance patient behaviour and potentially elevate the observed compliance and persistence rates. There is a strong preference, therefore, to study adherence in observational studies that more closely reflect daily clinical practise [40].

Different study approaches can be envisaged. It is preferred to perform prospective and comparative studies. Blinding is problematic, because double dummy studies will hamper the objective of the study. Retrospective studies are difficult to interpret because of the risk of confounding, since they cannot take into account all the differences in design between studies.

Studies that use databases in which pharmacy data are combined with hard clinical outcome data, such as fractures, will certainly be of value. In these studies, however, persistence but not compliance is measured, and have, therefore, their limitations.

Both direct and indirect methods for measuring adherence could be used (see Table 1). Direct methods are more expensive and more labour-intensive. Measurement of inert markers included in formulations is in theory a possibility but this is not generally accepted by authorities overseeing trials. The safety of the marker should be demonstrated and the marker has to be declared on the label. This could in turn influence the compliance. Indirect methods seem to be less accurate and reliable, but there exists a significant association between the two possibilities [41, 42]. For some active substances, such as strontium, direct measurements of drug or its metabolites in biological fluids may be used as validation of indirect measurement such as pill count.

More pragmatic is the use of indirect methods, but these methods should be improved. Self reporting by patients is in principle unreliable. At present, measurement of adherence in studies of osteoporosis relies mostly on pill counts and selfreporting [43]. Pill counts and self-reporting overestimate true compliance [44-49]. In addition, they provide no information on the patient's behaviour surrounding drug intake. Another possibility is the use of electronic monitoring devices with which patient's behaviour of drug intake can be closely monitored. Electronic monitoring of dose intake potentially offers a useful method for assessing adherence.

The best approach is to generate prospective data in an open comparative study. A number of problems need to be resolved when two different products are compared. Copayment could influence adherence. Another issue in a trial situation is that informed consent has to be signed by the patient. Thus the patient is aware of the objective of the study which may influence the result. Indeed, any prospective study in which adherence is a primary end-point is likely to overestimate adherence in clinical practice and

Table 1 Direct and indirect methods measuring compliance

\begin{tabular}{|c|c|}
\hline Direct methods & Indirect methods \\
\hline $\begin{array}{l}\text { Measurement of drug or metabolite } \\
\text { in a biologic fluid }\end{array}$ & $\begin{array}{l}\text { Self-reporting by the patient } \\
\text { (interview, diary, questionnaire) }\end{array}$ \\
\hline $\begin{array}{l}\text { Measurement of efficacy } \\
\text { surrogates (e.g., urinary excretion } \\
\text { of bone markers, BMD) }\end{array}$ & $\begin{array}{l}\text { Medication measurement (pill } \\
\text { count), refill logs (pharmacy) }\end{array}$ \\
\hline Direct intake observation & Electronic monitoring devices \\
\hline
\end{tabular}


may merely replicate information provided by intention-totreat analyses of phase III clinical trials. In such studies, persistence to therapy might be evaluated by measuring prescription refill dispensed to the patient over the study period and a patient questionnaire. An electronic pill counting device could also be used.

A totally different study format is to perform two singlearm open multi-centre studies each to investigate treatment satisfaction with therapy with two treatments $\mathrm{X}$ and $\mathrm{Y}$ as the primary objective. Scales of treatment satisfaction are now in development derived from quality of life (QoL) scales. Persistence to treatment and reasons for discontinuation are included as secondary objectives. Randomisation could be done at patient level. However, an interesting alternative possibility would be randomisation of the prescribing physician. This latter approach avoids a certain prescribing confounding and has the advantage of a more naturalistic design. The patient gets the prescription from his or her prescriber, and medicines are distributed by their own pharmacy. There are hardly extra visits necessary. At the end a meta-analysis in a pre-planned third study is done to compare the results of the two studies.

Cohort studies that examine persistence and compare results with historical data are prone to bias and are, thus, unreliable. For this reason, studies using retrospective data should be strongly discouraged.

Further investigation is also warranted to better understand the barriers to adherence with medical therapies and dietary and lifestyle interventions that are important for the optimal management of osteoporosis.

In conclusion adherence to therapy is a major problem in the treatment of osteoporosis. Both patient and medication factors are involved. Many stakeholders are interested in studying adherence with the objective of improving health outcomes. Study methods are not well developed at the moment and should be improved. Performing adherence studies will be stimulated when registration authorities accept the results of these studies and include the results in Sect. 5.1 of the SPC. At present, such data would only be included if a better outcome has been demonstrated. Reimbursement authorities might be convinced to consider this as important information for their reimbursement decisions. Adherence studies are an important aspect of outcome studies.

\section{References}

1. Caro JJ, Ishak KJ, Huybrechts KF et al (2004) The impact of compliance with osteoporosis therapy on fracture rates in actual practice. Osteoporosis Int 15:1003-1008

2. Huybrechts KF, Ishak KJ, Caro JJ (2006) Assessment of compliance with osteoporosis treatment and its consequences in a managed care population. Bone 38:922-928

3. Siris ES, Harris ST, Rosen CJ et al (2006) Adherence to bisphosphonate therapy and fracture rtes in osteoporotic women: relationship to vertebral and non-vertebral fractures from 2 US claims databases. Mayo Clinic Proc 81:1013-1022

4. Clowes JA, Peel NFA, Eastell R (2004) The impact of monitoring on adherence and persistence with antiresorptive treatment for postmenopausal osteoporosis: a randomised controlled trial. J Clin Endocrinol Metab 89:1117-1123

5. Yood RA, Emani S, Reid JI et al (2003) Compliance with pharmacological therapy for osteoporosis. Osteoporosis Int 14:965-968

6. Silverman S, Siris E, Abbott $T$ et al (2003) Adherence to bisphosphonate therapy is associated with decreased non-vertebral osteoporotic fracture risk. J Bone Miner Res 20 (suppl 1):S286

7. Weycker D, Macarios D, Edelsberg J et al (2006) Compliance with drug therapy for postmenopausal osteoporosis. Osteoporosis Int 17:1645-1652

8. Compston JE, Seeman E (2006) Compliance with osteoporosis therapy is the weakest link. Lancet 368:2005-2006

9. Miller NH (1997) Compliance with treatment regimens in chronic asymptomatic diseases. Am J Med 102:43-49

10. Willey C, Redding C, Stafford J et al (2000) Stages of change for adherence with medication regimens for chronic diseases: development and validation of a measure. Clin Ther 22:858-871

11. Donovan JL (1995) Patient decision making. The missing ingredient in compliance research. Int J Technol Assess Health Care 11:443-455

12. Kyngas H, Duffy ME, Kroll T (2000) Conceptual analysis of compliance. J Clin Nurs 9:5-12

13. Lutfey KE, Wishner WJ (1999) Beyond "compliance" is "adherence". Improving the prospect of diabetes care. Diabetes Care 22:635-639

14. Henneman EA, Lee JL, Cohen JI (1995) Collaboration: a concept analysis. J Adv Nurs 21:103-109

15. Madden BP (1990) The hybrid model for concept development: its value for the study of therapeutic alliance. ANS Adv Nurs Sci 12:75-87

16. Mullen PD (1997) Compliance becomes concordance. B Med J 314:691-692

17. McCombs JS, Thiebaudet P, McLaughlin-Milet C et al (2004) Compliance with drug therapies for the treatment and prevention of osteoporosis. Maturitas 48:271-287

18. Cooper A, Drake J, Brankin E, on behalf of the persist investigators (2006) Treatment persistence with once-monthly ibandronate and patient support vs. once weekly alendronate: results from the PERSIST study. J Clin Practice 60:896-905

19. Cramer JA, Amonkar MM, Hebborn A et al (2005) Compliance and persistence with bisphosphonate dosing regimens among women with postmenopausal osteoporosis. Curr Med Res Opin 21:1453-1460

20. Fardellone P, Gaudin AF, Cotte FE (2005) Comparison of the persistence of daily and weekly bisphosphonates in female patients treated for osteoporosis. J Bone Miner Res 20 (Suppl 1):S285-S286

21. Solomon DH, Avorn J, Katz et al (2005) Compliance with osteoporosis indications. Arch Intern Med 165:2414-2419

22. Docherty SM, Goodley A, Steel SA (2005) Compliance and effect of bone protective treatment in elderly females: 5 year follow-up study. Rheumatology 44(Suppl 1):134

23. Tosteson ANA, Grove MR, Hammond CS et al (2003) Early discontinuation of treatment for osteoporosis. Am J Med 115:209-216

24. Hamilton B, McCoy K, Taggart H (2003) Tolerability and compliance with risedronate in clinical practice. Osteoporosis Int 14:259-262

25. Prowse D, McGetrick V, Thompson AJ et al (2005) Persistence with oral therapy is high amongst patients followed in a DGH osteoporosis. Clinical Rheumatology 44(Suppl):135

26. Briesacher BA, Andrade S, Kahler K et al (2006) Economic impact of the previous year's adherence with bisphosphonates. J Bone Miner Res 21(suppl 1):S171

27. World Health Organization (2003) Adherence to long-term therapies: Evidence for action. WHO, Geneva, Switzerland 
28. Lamberg L (2000) Patient-physician relationship critical even during brief "medication checks". JAMA 284:29-31

29. Eraker SA, Kirscht JP, Becker MH (1984) Understanding and improving patient compliance. Ann Intern Med 100:258-268

30. Ammassari A, Trotta MP, Murri R et al (2002) Study group correlates and predictors of adherence to highly active antiretroviral therapy: Overview of Published. JAIDS 31(suppl 3):S123S127

31. McCloskey E, de Takats D, Orgee J et al (2005) Characteristics associated with non-persistence during daily therapy. Experience from the placebo wing of a community-based clinical trial. J Bone Miner Res 20(suppl 1):S282

32. Claxton AJ, Cranmer J, Pierce C (2001) A systematic review of the associations between dose regimens and medication compliance. Clin Ther 23:1296-1310

33. Thompson P, Cooper C, Carr A (2005) Factors influencing adherence to bisphosphonates for osteoporosis. J Bone Miner Res 20 (suppl):S394

34. Irvine J, Baker B, Smith J et al (1999) Poor adherence to placebo or amiodarone therapy predicts mortality: results from the CAMIAT study Psychosom Med 61:566-575

35. Granger BB, Swedberg K, Ekman I, for the CHARM investigators (2005) Adherence to candesartan and placebo and outcomes in chronic heart failure in the CHARM programme: double-blind, randomised, controlled clinical trial. Lancet 366:2005-2011

36. Delmas PD, Vrijens B, Eastell R et al (2007) Improving measurements of persistence on actonel treatment (IMPACT) investigators. Effect of monitoring bone turnover markers on persistence with risedronate treatment of postmenopausal osteoporosis. J Clin Endocrinol Metab 92:296-304

37. Cramer JA, Silverman S (2006) Persistence with Bisphosphonate Treatment for Osteoporosis: Finding the root of the problem. Am J Med 119:S12-S17

38. Kanis JA et al (2007) Glucocorticoid-induced osteoporosis: a systematic review and cost-utility analysis. Health Technol Assess $11: 1-256$
39. Stevenson M, Davies S (2006) DSU economic evaluation of pooled alendronate and risedronate compared with strontium ranelate, raloxifene, etidronate and teriparatide http://guidance. nice.org.uk/page.aspx?o=370643)

40. Spilker B (1991) Methods of assessing and improving patient compliance in clinical trials. In: Cramer JA, Spilker B (eds) (1991) Patient compliance in medical practice and clinical trials. Raven press, New York pp 37-56

41. Melnikow J, Kiefe C (1994) Patient compliance and medical research. Issues in methodology. J Gen Int Med 9:96-105

42. Turbi C, Herrero-Beaumont G, Acebes JC et al (2004) Compliance and satisfaction with raloxifene versus alendronate for the treatment of postmenopausal osteoporosis in clinical practice: An open-label, prospective, nonrandomized, observational study. Clin Ther 26:245-256

43. Farmer KC (1999) Methods for measuring and monitoring medication regimen adherence in clinical trials and clinical practice. Clin Ther 21:1074-1090

44. Straka RJ, Fish JT, Benson SR et al (1997) Patient self-reporting of compliance does not correspond with electronic monitoring: an evaluation using isosorbide dinitrate as a model drug. Pharmacotherapy $17: 126-132$

45. Lee JY, Kusek JW, Greene PG et al (1996) Assessing medication adherence by pill count and electronic monitoring in the African American Study of Kidney Disease and Hypertension (AASK) Pilot Study. Am J Hypertens 9:719-725

46. Mason BJ, Matsuyama JR, Jue SG (1995) Assessment of sulfonylurea adherence and metabolic control. Diabetes Educ 21:52-57

47. Paes AH, Bakker A, Soe-Agnie CJ (1998) Measurement of patient compliance. Pharm World Sci 20:73-77

48. Pullar T, Feely M (1990) Problems of compliance with drug treatment: new solutions? Pharm J 245:213-215

49. Schwed A, Fallab CL, Burnier M et al (1999) Electronic monitoring of compliance to lipid-lowering therapy in clinical practice. J Clin Pharmacol 39:402-409 